\section{Le posizioni di R. Guardini e di H. U. von Balthasar sulla questione della "fede di Gesù"**}

\author{
Ashraf N. I. Abdelmalak**
}

RECIBIDO: 16-03-17. APROBADO: 09-08-17

Riassunto: Negli ultimi anni il tema della fede "di" Gesù ha suscitato un grande interesse nella teologia sistematica. In questo breve studio saranno trattate le posizioni di due grandi teologi cattolici del XX secolo sulla questione della "fede di Gesù": quella del teologo italo-tedesco R. Guardini (1885-1968) e quella del teologo svizzero $H$. U. von Balthasar (1905-1985). Il presente articolo avrà, perciò, la struttura seguente: (1) Il dato biblico sulla fede di Gesù; (2) la fede di Gesù, la sua obbedienza e la sua solitudine secondo R. Guardini; (3) la fides Christi secondo H. U. von Balthasar. (4) Una breve conclusione.

Parole chiave: Gesù di Nazareth; fede; obbedienza; Guardini; von Balthasar.
The Thought of R. Guardini and H. U. von Balthasar on the Issue of the "Faith of Jesus"

Aвstract: During the past years, "Jesus' faith" has been a matter of interest to Sistematic Theology. This article analyzes the thought of two of the most recognized Catholic theologians of the twentieth century on this question: the ItalianGerman R. Guardini (1885-1968) and the Swiss H. U. von Balthasar (1905-1985). Its structure will then be the following: (1) Biblical data on the faith of Jesus; (2) the faith of Jesus, his obedience and his loneliness according to R. Guardini; (3) the fides Christi according to H. U. von Balthasar; (4) a brief conclusion.

KeY Words: Jesus of Nazareth; Faith; Obedience; Guardini; von Balthasar.

\section{PARA CITAR ESTE ARTÍCULO:}

Abdelmalak Ashraf N. I. "Le posizioni di R. Guardini e di H. U. von Balthasar". Theologica Xaveriana 185 (2018): 1-25. https://doi.org/10.11144/javeriana.tx68185.pgbs

\footnotetext{
${ }^{*}$ Questo articolo è frutto di una riflessione sviluppata all'interno di un seminario svolto nel ciclo dottorale all'Università Urbaniana a Roma, e di una ulteriore rielaborazione del suo contenuto nello svolgimento della docenza alla Universidad de San Buenaventura (Bogotá, Colombia).

** Dottore in Teologia dall'Università Urbaniana di Roma. Docente della Facultad de Ciencias Humanas y Sociales della Universidad de San Buenaventura (Bogotá, Colombia). OrCid 0000-0002-1958-3256. E-mail: ashrnaner3@hotmail.com
} 


\section{Introduzione}

Questa "fede del Figlio di Dio" [Gal 2,20] è certamente la fede dell'Apostolo delle genti in Gesù, ma suppone anche l'affidabilità di Gesù, che si fonda, sì, nel suo amore fino alla morte, ma anche nel suo essere Figlio di Dio [...]. Nella fede, Cristo non è soltanto Colui in cui crediamo, la manifestazione massima dell'amore di Dio, ma anche Colui al quale ci uniamo per poter credere. La fede, non solo guarda a Gesù, ma guarda dal punto di vista di Gesù, con i suoi occhi: è una partecipazione al suo modo di vedere. ${ }^{1}$

In tutta la storia del cristianesimo, si è parlato sempre e principalmente della fede "in" Gesù (Gesù il Cristo come "oggetto" della fede dei cristiani), ma negli ultimi anni il tema della fede "di" Gesù (Gesù come "soggetto" della fede) ha suscitato un grande interesse nella teologia sistematica. In questo breve studio saranno trattate le posizioni di due grandi teologi cattolici del XX secolo sulla questione della "fede di Gesù": quella del teologo italo-tedesco R. Guardini (1885-1968)² e quella del teologo svizzero H. U. von Balthasar (1905-1985). ${ }^{3}$

Per meglio comprendere le prese di posizione di R. Guardini e di H. U. von Balthasar sulla tematica della fede di Gesù, conviene partire dal dato biblico, in quanto sembra essere il fondamento principale sul quale si basano tanto Guardini quanto von Balthasar per affrontare il tema della fede di Gesù e della sua obbedienza. Una volta fatto ciò, si presenta brevemente il pensiero guardiniano sul tema; ed infine, si analizza la tesi proposta da H. U. von Balthasar sulla Fides Christi, in quanto quest' ultimo conosce fino in fondo il pensiero guardiniano nel suo complesso $\mathrm{f}^{4}$, prende

\footnotetext{
${ }^{1}$ Francesco I, "Lettera enciclica Lumen fidei (29 giugno 2013)", 17, 18.

${ }^{2}$ Romano Guardini è nato a Verona (Italia), nel 1885; ed è morto in Germania, nel 1968. Per una autobiografia, cfr. Guardini, Apuntes para una autobiografia. Per ulteriori informazioni sulla vita e sulla opera di Guardini, cfr. principalmente Gerl, Romano Guardini: La vita e l'opera; ed anche, Sommavilla, "Introduzione a R. Guardini", 123-132; Engelmann-Ferrier, "Introduzione a Romano Guardini”; Mondin, I grandi teologi del secolo ventesimo. I teologi cattolici, Vol. 1, 89-120; von Balthasar, Romano Guardini: riforma dalle origini; Gibellini, La teología del siglo XX, 233-239; López Quintás, Romano Guardini: maestro de vida; ídem, "Estudio introductorio a R. Guardini”, xvii-xxxiii; ídem, La verdadera imagen de Romano Guardini, 41-53; Bueno de la Fuente, "Guardini, Romano", 460-471.
}

${ }^{3}$ Hans Urs von Balthasar è nato a Lucerna (Svizzera), nel 1905; ed è morto nel 1988. Per ulteriori informazioni sulla vita e sulla opera di von Balthasar, cfr. Mondin, I grandi teologi del secolo ventesimo 1: I teologi cattolici, 267-296; ídem, La nuova teologia cattolica: Da Karl Rahner a Urs von Balthasar, 165-178; Communio, ed. española, núm. IV (1988); González de Cardedal, "La obra teológica de Hans Urs von Balthasar", 365-396; Henrici, "Semblanza de Hans Urs von Balthasar”, 356-391; Lehmann e Kasper (edd.), Hans Urs von Balthasar: figura e opera; Scola, Hans Urs von Balthasar: un estilo teológico; Gibellini, La teología del siglo XX, 254-270; Sayés, La esencia del cristianismo: diálogo con K. Rahner y H. U. von Balthasar, 207-375; Guerriero, Hans Urs von Balthasar.

${ }^{4}$ Von Balthasar, Romano Guardini. 
in considerazione la posizione guardiniana sulla fede di Gesù, e la include nella sua posizione, anche se con l'intenzione di superarla (Aufhebung). Il presente articolo avrà, perciò, la struttura seguente: (1) Il dato biblico sulla fede di Gesù. (2) La fede di Gesù, la sua obbedienza e la sua solitudine secondo R. Guardini. (3) La fides Christi secondo H. U. von Balthasar. (4) Una breve conclusione.

\section{Il dato biblico sulla fede di Gesù}

\section{II mistero della fede}

Definire il "credere" o la "fede" è un compito complesso, perché il mistero della fede non si lascia rinchiudere in una definizione precisa che esprima sufficientemente la sua naturas'. Per la Bibbia la "fede" è la fonte di tutta la vita religiosa; è l'unico atteggiamento religioso dell'uomo che è svegliato e confermato dal comportamento unico e unitario del Dio fedele (cfr. Dt 7,9; cfr. Is 49,7) con l'uomo; è anche una delle linee fondamentali del Vangelo. Questa parola (fede), infatti, significa biblicamente il totale riferimento da parte dell'uomo a Dio, conosciuto nella rivelazione; essa indica la risposta umana al progetto del Dio fedele nella storia. L'atteggiamento della fede si converte nell'atteggiamento semplicemente necessario per l'esistenza dell'uomo (cfr. Is 7,9; Rm 9-10). Tale uomo che crede si scopre aperto alla trascendenza e dotato di una libertà e di una responsabilità.

\section{La fede nell'Antico Testamento e nel Nuovo Testamento}

Innanzitutto, la terminologia veterotestamentaria fa vedere la fede nelle sue dimensioni di fiducia, conoscenza e obbedienza, in quanto la radice fondamentale ( $m n$ ) indica stabilità e sicurezza derivanti dell'appoggiarsi totalmente nell'altro, e batah indica la sicurezza, la fiducia. E tali atteggiamenti, poi, vengono chiariti nell'Antico Testamento con tanti altri gruppi etimologici (chasah, yichel... etc.). Fede vuole dire, dunque, fidarsi ed abbandonarsi nelle mani di Dio (cfr. Gn 15,6). Nella tradizione israelita, infatti, si conosce e si esige un assoluto ed esclusivo abbandono a Yahvé; e questa fiducia senza riserve in Dio include la speranza di salvezza (cfr. Gb 11,18) e la fede nel Dio dei poveri (cfr. Sal 22,40). In quest'ottica, la terminologia dell' Antico Testamento descrive la fede come "conoscenza-riconoscenza" di YHWH e del suo potere salvifico e dominante rivelato nella storia.

${ }^{5}$ Per parlare qui della fede nell' Antico Testamento e nel Nuovo Testamento, si serve principalmente della seguente bibliografia: Marconcini, "Fe", 653-664; Duplacy, "Fe”, 327-335; Nieto, "Fe", 436-442; Dheilly, "Fe", 445-453; Wildberger, "Firme, seguro", 276-319; Gerstenberger, "Confiar”, 439-446; Barth,

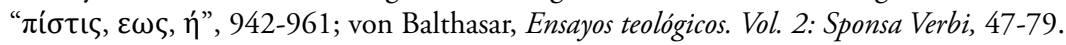


La varietà della terminologia dell'Antico Testamento, poi, si concentra in un unico termine, frequentissimo, del Nuovo Testamento, ovvero il verbo pistéuō (credere) ed il sostantivo pistis (fede). Credere o avere fede, secondo il Nuovo Testamento, è riconoscere Gesù, mediante la sua morte e risurrezione, come il Messia, a tal punto che il cristiano viene qualificato semplicemente come "il credente" (cfr. Eb 2,44; 4,32; 11,21). Questi termini (credere e fede), i più usati nel Nuovo Testamento (243 volte ciascuno $)^{6}$ dopo termini come Dio, Cristo, Signore, Gesù e Spirito, ha conosciuto una evoluzione, ovvero di passare da un "senso soggettivo" (l'atto di credere: abbandono, timore, riverenza, culto, obbedienza, amore, fiducia, fedeltà, speranza... etc.) a un "senso oggettivo" (il contenuto che si crede o il deposito della fede).

In questo senso, lo studio del vocabolario biblico rivela che la fede ha due poli: la fiducia che si dirige a una persona "fedele" e che implica l'uomo intero (credere in qualcuno); e il processo della intelligenza (credere in qualcosa). In altri termini, il Nuovo Testamento parla della pistis (pistéuein) eis e della pistéuein hoti, espressioni che la riflessione teologica successiva tradurrà in fides qua (dimensione fiduciale) e fides quae (dimensione intellettuale) ${ }^{7}$.

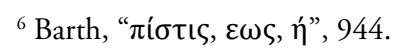

${ }^{7}$ Alszeghy-Flick, Come si fa teologia: Introduzione allo studio della teologia dogmatica, 18: "Perciò la formula credere in, che esprime l'orientamento personale e dinamico della fede, fin dai sinottici è accompagnata dalle formule credere che e credere a», che si riferiscono ad asserti accettati come veri perché 'detti' da Dio. L'aspetto intellettuale della fede non è l'unico, né il principale, ma è indispensabile per l'esistenza cristiana, ed è l'orizzonte di ogni studio teologico". San Tommaso d'Aquino, dal suo canto, parla di tre formule dello stesso ed unico atto della fede, che ha una relazione distinta con l'oggetto di fede: credere in (l'oggetto dal punto di vista finale o l'atteggiamento esistenziale verso Dio), credere che (l'oggetto dal punto di vista materiale o l'affermazione da credere) e credere a (l'oggetto dal punto di vista formale o l'autorità di Dio). Al riguardo, cfr. Santo Tomás de Aquino, Suma de teología, Tomo III, p. II-II, q. 2, a. 2. In questo senso, F. Hadjadj osserva: "Tommaso d'Aquino è più preciso nella sua terminologia. Alla distinzione che instaura tra il credere in Deum (credere in/verso Dio), e credere Deum (credere che Dio è Dio), aggiunge quella del credere Deo (credere a Dio). Nel primo caso si tratta dell'oggetto della fede considerato dal punto di vista del fine, come quello che realizza la beatitudine. Nel secondo caso, l'oggetto della fede è considerato dal punto di vista materiale, ovvero in quanto questo o quell'articolo proposto si rapportano a Dio. Nel terzo caso, l'oggetto della fede è considerato dal punto di vista formale, come ciò che lo motiva, cioè l'autorità di Dio che si rivela. Se nel primo caso l'oggetto della fede, percepito come il bene supremo, mette in movimento la volontà (credere in Deum), negli altri due casi l'oggetto della fede concerne l'intelligenza e si presenta come ciò a cui io credo (quod creditur), e ciò tramite cui io credo (quo creditur). Però, per ciò che concerne il quo creditur, il motivo della fede dei demoni non è il medesimo di quello della fede teologale. Il credere Deo (dativo) è più un gesto di fiducia. È un credere a patire dal Dio che si rivela: è l'eterno stesso che illumina l'intelligenza e la porta ad accogliere una rivelazione che va ben oltre le sue forze naturali. Ma i demoni, soprattutto, non vogliono nulla che li sovrasti e li ecceda, tanto sul versante della volontà quanto sul versante dell'intelligenza" (Hadjadj, La fede dei demoni: Ovvero il superamento dell'ateismo, 61-62). 
In definitiva, se nell'Antico Testamento, parlando della fede, si evidenza più l'aspetto della fiducia, nel Nuovo Testamento si esalta più l'aspetto di assenso al messaggio cristiano. La fede deve, comunque, avere tre elementi fondamentali, senza i quali non c'è una fede vera: obbedienza (ascoltare), fiducia (abbandonarsi) e fedeltà (accogliere un impegno). La fede, pertanto, si realizza nell'obbedienza, si vive e si mantiene nella fiducia e nella fedeltà, e si manifesta nell'amore operativo. L'incredulità (apistía, apeíseia) è, infine, un peccato di autosufficienza. La persona incredula si appoggia unicamente nelle sue proprie forze e sui propri valori, invece di appoggiarsi in Dio e abbandonarsi a Lui. Tale peccato, poi, secondo il Nuovo Testamento, ha un carattere cristologico, in quanto la persona incredula non crede in Gesù Cristo (cfr. Mc 16,16; Gv 3,18).

Il Nuovo Testamento parla chiaramente, inoltre, del credere e della fede "in Gesù". Credere "in Gesù" è una cosa propria dei suoi discepoli; e la fede in lui unisce i suoi discepoli con Lui e tra di loro, facendo condividere loro il segreto della sua persona. In quest'ottica, la fede in lui e nella sua parola è, già nella vita terrena di Gesù, il vincolo più prezioso per i suoi discepoli (la comunità dei poveri di YHWH). Quando Gesù, il servo, arriva a Gerusalemme per obbedire fino alla morte (cfr. Flp 2,7s.), con la sua morte e risurrezione, porta alla "perfezione" la fede (cfr. Eb 12,2) dei poveri, mostrando una fiducia assoluta in colui che poteva, mediante la risurrezione, "salvarlo della morte" (cfr. Eb 5,7). Grazie all'evento pasquale, dunque, la fede dei discepoli di Gesù fa un passo decisivo per arrivare ad essere la fede della Chiesa. Mediante la fede pasquale, infatti, i discepoli, testimoni di ciò che ha fatto e detto Gesù (cfr. 1 Gv 1,1-4), proclamano quest'ultimo come "Signore e Cristo" (cfr. Atti 10,39); e la loro fede in Lui diventa capace di andare "fino al sangue" (cfr. Eb 12,4).

Da tutto ciò risulta che credere, per il Nuovo Testamento, è, innanzitutto, accogliere questa predicazione dei testimoni (il vangelo e la parola), confessando che Gesù è il Cristo, il Signore (cfr. 1Cor 12,3; Rm 10, 9; 1Gv 2,22). La fede "in Gesù", morto e risorto, che è un dono divino, è la sorgente della salvezza (cfr. Mc 5,34; Lc 8,48) e della vita eterna (cfr. Gv 6,47); tale fede è necessaria per la salvezza (cfr. Gv 3,18.36) e per entrare a fare parte del regno di Dio (cfr. Mc 1,15).

La fede della quale parla il Nuovo Testamento, in somma, è fondamentalmente la fede "in Gesù", il Figlio di Dio divenuto uomo, crocifisso e risorto; e credere in Dio ed in Gesù è la stessa cosa, poiché Gesù e il Padre sono uno (cfr. Gv 12,44; 10,30;

\footnotetext{
${ }^{8}$ Marconcini, "Fe", 653-664; Duplacy, "Fe", 327-335; Nieto, "Fe", 436-442; Dheilly, "Fe", 445-453. Per altri elementi connessi alla questione della "fede", come la gnosi/conoscenza (credere e conoscere), la visione (credere e vedere), le opere (credere e operare) ed il dono (credere quale opera-dono divino ed opera-ricerca umana), si può consultare Marconcini, "Fe", 664-671.
} 
17,21). L'oggetto della fede neotestamentaria è, quindi, Gesù Cristo, in quanto Egli è la "luce" (cfr. Gv 12, 16.36), il "YHWH-Io sono" o Dio (cfr. Gv 13,19; 8,24), il "Signore" (cfr. 1Cor 12,3), il "Figlio" del Padre (cfr. Gv 14,10-11; 10,1), il "Messia" (cfr. Mt 11,27; Mc 9,6; Gv 20, 31), il "morto-risorto" (cfr. 1Cor 15)... etc. Il Vangelo di Gesù Cristo consiste propriamente nel credere (in Lui) ed amare (gli uni gli altri), come dice $(1 \mathrm{Gv} 3,23)$. Dio è certamente la fonte della fede; Egli si serve, però, di cause seconde per portare l'uomo alla fede nel suo Figlio, Gesù Cristo: i miracoli (cfr. Gv 10,38; 11, 47-48), la testimonianza (cfr. Gv 1,7; 8,18; 12,17; 15,26; 19,35) e la parola (cfr. Gv 15,47; Rm 10,17) .

\section{Fede in Gesù o fede di Gesù?}

Ora, le domande che sorgono sono le seguenti: parla il Nuovo Testamento, non solo della fede "in" Gesù, ma anche della fede "di" Gesù? Gesù stesso aveva fede o richiedeva soltanto la fede in Lui, come lo richiedeva per il suo Padre? Come si è visto, tanto l'Antico Testamento quanto il Nuovo Testamento parlano chiaramente del Dio fedele alla sua parola (cfr. Dt 7,9; 1Tes 5,24); e tale Dio fedele darà la corona della vita alla persona fedele fino alla morte (cfr. Ap 2,10). La fides e la fidelitas di Dio, il suo essere fedele all'alleanza, poiché è fedele a se stesso, sono il fondamento della forma specificamente umana della fides, che, a sua volta, è quella fedeltà che si riposa nella riconoscenza di Dio in quanto Dio e, per conseguenza, nella perfetta fiducia in Lui ${ }^{10}$.

Gesù Cristo è, poi, la fedeltà stessa e il suo nome è "il fedele" (cfr. Ap 19,11). Egli è fedele davanti Dio (cfr. Eb 2,17) e davanti agli uomini (cfr. 2Tim 2,13). Nella Bibbia, tanto nell' Antico Testamento come nel Nuovo Testamento, si trovano, inoltre, tanti altri modelli di fede: Israele ("il popolo della fede" grazie all'alleanza, all'elezione e alla promessa); i testimoni dell'Antico Testamento ("gli uomini della fede" [cfr. Eb 12]); Abramo ("il padre della fede" e il "paradigma del credente"); Maria Vergine ("la credente"); i poveri e i piccoli; i discepoli... etc.; ed anche, e fondamentalmente, Gesù Cristo. Secondo E. Martín Nieto, si potrebbe sostenere biblicamente che Gesù è "il credente" ${ }^{11}$.

Gesù Cristo è, senza dubbio, il grande modello, l'unico, della fede. Possiamo definirlo come "Il Credente", con l'articolo e con la maiuscola, l'incomparabile

\footnotetext{
${ }^{9}$ Duplacy, "Fe", 331-335; Marconcini, "Fe”, 667-668; Nieto, "Fe”, 436-442.

${ }^{10}$ Wildberger, "Firme, seguro", 276-319; von Balthasar, Ensayos teológicos 2, 54.

${ }^{11}$ Nieto, "Fe", 438-441. Della possibilità o meno di basarsi sulle affermazioni bibliche per sostenere la fede "di”" Gesù, si parlerà successivamente quando si presenta la tesi di von Balthasar a proposito della fides Christi.
} 
paradigma della fede. Crede nel suo Padre, si fida di lui, lo ascolta e gli obbedisce (Gv 15,15), compie i suoi comandamenti e rimane nel suo amore (Gv 15,15). Fa sempre quello che gli [al Padre] fa piacere $(\mathrm{Gv} 8,29)$. Ha manifestato la sua obbedienza assoluta al Padre accettando la morte, ha sopportato la croce assumendo coraggiosamente la ignominia (Eb 12,2) e mettendosi, alla fine, fiduciosamente nelle sue mani (Lc 23,46). ${ }^{12}$

\section{La fede di Gesù, la sua obbedienza e la sua solitudine secondo R. Guardini ${ }^{13}$}

\section{La fede di Gesù}

La fede è uno dei temi centrali nel pensiero di Guardini, al quale ha dedicato tante riflessioni ${ }^{14}$; ma la questione che ci interessa ora è la fede in rapporto a Gesù. Che tipo di relazione -si domanda Guardini- ha Gesù rispetto alla fede? Fu Gesù stesso un credente? Quando Gesù parla del Padre, parla per fede? Partendo dal Nuovo Testamento, la risposta più spontanea del nostro autore suona così: no, Gesù non fu un credente. Le ragioni profonde di tale precisa affermazione guardiniana possono essere sintetizzate nelle seguenti osservazioni. Anzitutto, l'atteggiamento di Gesù nei confronti di Dio, come parla di Lui e come si situa davanti a lui, dice che Gesù non era credente.

La fede, poi, significa stabilire un ponte con la realtà divina, ed abbracciare tale realtà e vivere di essa. E ciò vuol dire una trasposizione e trasformazione della propria esistenza nella direzione di quella realtà, partendo da essa stessa; e che quella realtà viene dall'alto, e ciò che c'è nell'uomo viene dal basso, che spesso fa resistenza a tale realtà. La fede indica sempre, quindi, una lotta rinnovata dalla fede, una tensione, una prova ed una costanza fino a raggiungere nuovamente una sicurezza ed a passare sempre di nuovo dalla pistis (fiducia della fede) alla gnosis (conoscenza della fede). Ora, tutto questo non si trova in Gesù. Gesù non passa, infatti, dal non credere al credere; e non si vede in Lui che una prima vita infantile di fede sia stata attraversata dalla crisi, dalla quale è scaturita una fede rinnovata e fortificata. In Gesù non si parla né di prove o tentazioni contro la fede, né di lotta e vittoria della fede. In lui, non si trova assolutamente l'atteggiamento della fede, che consiste nel fatto che l'uomo abbraccia

\footnotetext{
${ }^{12}$ Ibid., 440. La traduzione è nostra.

${ }^{13}$ A proposito del metodo filosofico-teologico, delle opere e della cristologia di Guardini, cfr. Abdelmalak, "Haciéndose obediente hasta la muerte, y muerte de cruz": R. Guardini y H. U. von Balthasar ante el sentido teológico de la muerte en cruz de Jesucristo, 42-48.

${ }^{14}$ Una presentazione guardiniana integrale della fede nella sua genesi, nel suo rapporto con la conoscenza, l'azione, l'amore e la speranza, nelle sue crisi e nelle sue forme, e della relazione tra la fede e la Chiesa (i dogmi ed i sacramenti), si trova in Guardini, La vita della fede. Cfr. anche idem, L'esistenza e la fede; idem, La fe en nuestro tiempo; idem, Fede - religione - esperienza: saggi teologici.
} 
una nuova realtà che gli viene incontro, né una lotta-resistenza da parte dell'“antica” realtà contro quella nuova. Egli ha ciò che dice e possiede Dio del quale parla; e qui non c'è nessuna dualità, ma unità. Tutte le prove e le tentazioni che Gesù doveva affrontare non toccavano la realtà della fede. La chiara conclusione guardiniana è, dunque, che Gesù non fu un credente, né un illuminato, né un profeta; Egli ha ciò di cui parla, ed il Dio del quale parla è in Lui. In definitiva, Egli è il mistero del Dio-uomo ${ }^{15}$.

Il nostro autore rifiuta, insomma, ogni teoria che afferma che Gesù, uomo come noi, abbia cercato la sua salvezza; e che la sua vita abbia la stessa direzione come la nostra: dall'umano a Dio. In quel caso, sì, di conseguenza, Egli sarebbe anche un credente; e avrebbe istituito formalmente l'atteggiamento credente del cristiano e avrebbe dato l'esempio di esso. Ora, nonostante la sua consapevolezza del fatto che tale teoria ha qualcosa di grande, ovvero essa prende sul serio ciò che è cristiano, il nostro autore osserva che in questa teoria-concezione, non si dà una redenzione reale, e con ciò cade il più profondo del cristianesimo: Gesù è il redentore, il mediatore ${ }^{16}$.

In Gesù Cristo, afferma Guardini, non si trova la ricerca religiosa, come commozione nell'incontro col sacro, come conversione e cambiamento di vita. Dal centro del suo essere, invece, proviene qualcosa del tutto singolare, ossia la realtà di "Dio". Il Dio di cui parla l'Antico Testamento è presente in lui. In questo senso, il nostro autore preferisce parlare di un' "obbedienza" piuttosto che di una "fede" di Gesù. Gesù il Cristo, perciò, non crede, ma rende possibile il credere. Egli non prova il sentimento religioso, ma lo accende, lo suscita. Il suo atteggiamento verso Dio è diverso da quello degli uomini; Egli, cioè, non tende come gli uomini verso Dio, Padre e creatore di tutti, ma rende possibile la realtà che il volto del Padre appaia e si manifesti ad essi, facendoli così entrare in dialogo con $\mathrm{Dio}^{17}$.

La fede non è un atto qualsiasi, ma è da parte dell'uomo la risposta alla venuta del Regno di Dio. Così la fede intesa bene è il contenuto stesso dell'invito fatto da Gesù. Egli però in persona non ha la "fede". Per lui la parola è senza oggetto. Egli non è là dove si crede, è la dove la fede si dirige. Più esattamente: Egli rende possibile la fede. ${ }^{18}$

Tutti gli uomini sono chiamati a credere nel Dio di Gesù Cristo, ma "Cristo stesso non "crede". Egli esiste come colui che è, ovvero il Figlio di Dio. Credere

\footnotetext{
${ }^{15}$ Idem, Jesucristo: palabras espirituales, 61-67.

${ }^{16}$ Ibid., 62-63.

${ }^{17}$ Idem, La realtà umana del Signore: saggio sulla psicologia di Gesù, 99-100.

${ }^{18}$ Ibid., 98.
} 
significa avere parte a ciò che Cristo è, non nel credere, ma nell'essere ${ }^{19}$. Guardini, quindi, non parla della fede che poteva avere Gesù in Dio, perché la fede, ancora una volta, "è un movimento da parte dell'uomo, che corrisponde al movimento della venuta del Salvatore" ${ }^{20}$; ma egli parla piuttosto dell'obbedienza di Gesù verso il Padre e la relaziona alla sua identità filiale e alla sua libertà. La sua obbedienza alla volontà del Padre, di fatto, collima con questa volontà, fino ad identificarsi con essa. Gesù è definitivamente e per sempre "Figlio"; però, proprio per questo è perfettamente libero e realizza completamente se stesso. In lui non si trova alcuna traccia di immaturità o di ribellione, di debolezza o di rancore, di morbida soggezione o di gelosia. Egli è colui che obbedisce, come il Padre è colui che comanda ${ }^{21}$.

L'obbedienza di Gesù come tale è pari al comando che Egli riceve. Essa non proviene dal rapporto di una persona più debole di fronte ad una più forte, o dal confronto della realtà di fatto con la norma; ma essa, proprio come obbedienza, è altrettanto forte e altrettanto valida quanto al comando. L'obbedienza di Gesù è il bene che viene eseguito, come l'ordine del Padre è il bene che viene comandato; nel più intimo sono una cosa sola. ${ }^{22}$

\section{L'obbedienza di Gesù, la sua interiorità e la volontà del Padre}

In questo contesto, dunque, la questione della "volontà del Padre" e della "libertà di Gesù”, in riferimento all'obbedienza di Gesù nel pensiero guardiniano, merita di essere trattata un po' da vicino. Innanzitutto, Guardini dice che il cammino del destino di Gesù e del modo in cui Egli porta a compimento la sua missione non si possono ricondurre a "motivi", dal momento che l'ultimo "perché" viene dall'insondabilità di ciò che Egli chiama esplicitamente "volontà del Padre" ${ }^{23}$. Egli, di fatto, parla continuamente della volontà del Padre. Non possiamo, però, immaginare il volere del Padre come una serie di indicazioni e di comandi fissati a priori, che contengono tutto ciò che si deve compiere nel corso del tempo. "La volontà del Padre è invece qualcosa che vive in Gesù, si sviluppa e si definisce man mano che la storia procede. È il Padre stesso nell'esercizio del suo volere, che 'è presso di Lui sempre"”24.

\footnotetext{
${ }^{19}$ Idem, Il Signore: Riflessioni sulla persona e sulla vita di Gesù Cristo, 263.

${ }^{20}$ Idem, La realtà umana del Signore, 153.

${ }^{21}$ Ibid., 103-108.

${ }^{22}$ Ibid., 109.

${ }^{23}$ Idem, Il Signore, 18.

${ }^{24}$ Ibid., 61.
} 
La volontà del Padre è, in ultima analisi, l'amore del Padre. Essa eleva Gesù all'intimità amorosa con Dio. Questa volontà, che di ora in ora si realizza in precetti, è l'amore dello Spirito Santo. Di volta in volta, da essa scaturisce ciò che Gesù fa ${ }^{25}$. La volontà di Gesù, poi, si accorda perfettamente con quella del Padre, il quale guida la storia sacra e determina l'ora di ciascun avvenimento. "La volontà del Padre è per lui nello Spirito ciò che nella vita naturale è il sangue" ${ }^{27}$. In realtà, "consegnatosi ultimamente alla volontà del Padre, il Figlio può essere esteriormente sopraffatto dal male e crocifisso, ma in fondo Egli non combatte affatto contro il male, lo giudica soltanto" 28 .

A questo punto, però, ci si può giustamente interrogare sulla libertà di Gesù e sulla sua posizione davanti alla volontà del Padre. Al riguardo, il nostro autore sostiene che quel che richiede la volontà del Padre è ciò che la volontà del Figlio desidera realizzare liberamente. In effetti, "ciò che si esige da questo cuore (di Gesù), è al tempo stesso ciò cui aspira da tutte le radici del suo essere, e in cui si trova il suo più limpido adempimento" ${ }^{29}$. La volontà del Padre, dunque, ha guidato l'azione di Gesù non come programma, ma come energia viva, il cui contenuto si dischiudeva man mano in ogni nuova situazione $e^{30}$. Egli è, di fatto, una cosa sola con la volontà del Padre. Egli sta dentro questa volontà, vi si attiene ${ }^{31}$.

A proposito della libertà di Gesù, Guardini, quindi, ritiene che Egli è perfettamente libero. Egli è libero da tutte le brame, da ogni paura per il possesso o il vitto, da qualsiasi risentimento, sia pure il più occulto, contro ciò di cui non gode. In lui, di fatto, la libertà è del tutto naturale ${ }^{32}$. In realtà, "la libertà di Cristo deriva dal fatto che Egli è interamente entro l'amore di Dio; e il suo orientamento spirituale è la volontà di salvare il mondo, sostenuta dalla serietà (del decreto) di Dio" ${ }^{33}$.

In sintesi, Gesù di Nazareth non crede, ma obbedisce e vive dal di dentro la realtà di Dio. Egli non cerca, non discute e non dubita; afferma come colui che sa. Perciò, paternità e provvidenza di Dio non sono oggetto di problema, ma di rivelazione.

\footnotetext{
${ }^{25}$ Ibid., 62.

${ }^{26}$ Idem, La realtà umana del Signore, 48.

${ }^{27}$ Idem, Il Signore, 150.

${ }^{28}$ Von Balthasar, Romano Guardini, 105.

${ }^{29}$ Guardini, Il Signore, 149.

${ }^{30}$ Ibid., 153.

${ }^{31}$ Ibid., 192.

${ }^{32}$ Ibid., 371.

${ }^{33}$ Ibid., 405.
} 
Egli non esamina né analizza, ma "annunzia qualcosa che ancora non c'è ma che però deve realizzarsi, cioè il regno di Dio"34. Guardini collega così la "volontà del Padre" e la "libertà del Figlio" per parlare dell'obbedienza assoluta di Gesù, e non della sua fede. In effetti, egli afferma: "nel Figlio eterno, con il suo entrare nella storia, la sua umiltà si è fatta disponibile all'obbedienza assoluta (Eb 10, 5-9)" ${ }^{35}$.

Per raggiungere una visione profonda dell'interiorità di Gesù, si deve, in ultima analisi, partire dal significato della "volontà" del Dio-Padre nella sua vita e dalla sua attenzione fedele a tale volontà; poiché propriamente in questo si discopre il vincolo tra obbedienza ed autenticità personale. Tutta la vita del Signore è, infatti, una vita guidata dalla volontà del Padre nello Spirito; e giustamente per non fare la sua propria volontà, ma quella del Padre, Gesù compie anche la sua volontà. La volontà del Padre è l'amore del Padre; è lui stesso. Nella sua volontà, il Padre viene a Gesù. Il suo chiamare, il suo comandare ed il suo esigere, è un venire. E nell'accettazione di tale volontà e tale venire, Gesù riceve il Padre stesso. In definitiva, se si parte dalla volontà del Padre, ci si può avvicinare adeguatamente all'interiorità di Gesù, alla sua obbedienza ed alla sua libertà, e percepire anche un eterno dialogo intimo ed una armonia perfetta tra il Padre ed il Figlio pienamente umanizzato nel loro Spirito ${ }^{36}$.

\section{La solitudine di Gesù}

In relazione alla questione della psicologia di Gesù in generale ${ }^{37}$, a quella della sua obbedienza-libertà ed a quella della fede di Gesù, Guardini tratta anche il tema della solitudine unica di Gesù ${ }^{38}$. Secondo il nostro autore, Gesù Cristo non ha avuto, in realtà, un reale amico, perché la vera amicizia chiede la parità tra gli amici ${ }^{39}$. Nel caso di Gesù "come avrebbe potuto qualcuno anche solo porsi accanto a lui in quella parità di grado che appartiene alla vera amicizia, a lui, che veniva dal Padre occulto, che recava in sé il senso del mondo e aveva assunto su di sé la responsabilità per la sua salvezza?" 40 . Tuttavia, la solitudine di Gesù non deriva dalla mancanza di un vero

${ }^{34} \mathrm{Idem}$, La realtà umana del Signore, 45.

${ }^{35}$ Idem, Il Signore, 725.

${ }^{36}$ Idem, Jesucristo, 71-76.

${ }^{37}$ Cfr. principalmente idem, La realtà umana del Signore; idem, Il Signore.

${ }^{38}$ Idem, Libertad, gracia y destino, 180.

${ }^{39}$ Idem, Il Signore, 253-254: "E sebbene Egli abbia anche detto ai suoi nell'ora del congedo: 'Non vi chiamo più servi [...]. Vi chiamo amici' (Gv 15,15), ciò significa appunto un dono del suo amore, non l'espressione di un rapporto che andasse da loro a Lui".

${ }^{40}$ Ibid., 253. 
amico o dall'abbandono della gente, infatti attorno a Gesù c'era sempre gente (gli Apostoli o altre persone). Egli è solo anche se è circondato da persone. "Non è che Egli si trovi solo semplicemente di fatto, è una solitudine qualitativa, assoluta..." ${ }^{41}$.

Per comprendere bene, poi, la solitudine di Gesù, si deve tenere presente lo svolgimento della missione e della vicenda di Gesù. Attorno a Gesù, infatti, si trovava uno spazio ininterrotto di solitudine estrema a causa dell'incomprensione da parte degli uomini della sua persona e della sua missione. Nessuno lo capiva fra quanti gli stavano accanto ${ }^{42}$. In questo senso, si può dire che "Gesù durante la sua vita è stato intimamente solitario. Nessuno gli è stato accanto realmente. Nessuno ha condiviso i suoi pensieri e nessuno lo ha aiutato nella sua opera" ${ }^{\text {43. }}$

In realtà, la solitudine, per così dire, ha accompagnato Gesù nell'intera sua esistenza. Da quando Egli era un fanciullo ${ }^{44}$ fino alla sua morte ${ }^{45}$, anche le persone più vicine a Lui dimostravano una situazione d'incomprensione nei suoi confronti. Mentre Gesù svolgeva il suo annuncio, la solitudine indicibile appariva più chiara e dura. Necessariamente, quindi, deve essere stata terribile la solitudine nella quale Gesù di Nazareth viveva: la solitudine del Figlio di Dio messo in catene dall'uomo ${ }^{46}$.

Da notare che il nostro autore non parla di un preciso periodo in cui Cristo ha vissuto la sua solitudine estrema, ma dice che tutta la sua vita-esistenza è stata attraversata da tale realtà. Nell'ora della sua morte, però, Egli ha sperimentato tale solitudine in modo del tutto singolare. Egli si pone interamente nell'"ora" ed era "totalmente nella solitudine estrema, con il peccato che gli è stato addossato, al cospetto della giustizia di Dio" ${ }^{47}$. Ma la domanda che sorge allora è la seguente: se Cristo in sé, in quanto l'Uomo-Dio, è incomprensibile alla mente umana, allora la sofferenza provocata dalla sua solitudine è causata soltanto dall'incomprensione della gente?

Per rispondere a tale questione, si deve evidenziare un altro aspetto della solitudine di Gesù sottolineato da Guardini, ovvero che essa non è causata soltanto dal fatto della non comprensione da parte degli uomini, ma che è anche legata alla sua coscienza. Egli conosce la perdizione del mondo, grazie alla sua conoscenza e alla sua relazione con il Padre. "Onde la spaventosa chiarezza di tale sapere.

\footnotetext{
${ }^{41}$ Idem, La realtà umana del Signore, 134.

${ }^{42}$ Ibid., 58.

${ }^{43}$ Idem, Il Signore, 93.

${ }^{44}$ Ibid., 93.

${ }^{45}$ Ibid., 468-529.

46 Ibid., 233.

${ }^{47}$ Ibid., 32.
} 
Onde anche la sua infinita solitudine. È realmente il veggente tra ciechi, colui che ha sensibilità tra ottusi, chi è libero e nell'ordine tra confusi” ${ }^{48}$. Per questo Egli è così inesorabilmente solo. È stato sempre un sofferente e sarebbe stato tale anche se gli uomini avessero accolto nella fede e nell'amore il suo messaggio. La sua vita, infatti, è stata di una sofferenza così profonda che ad essa non s'avvicina alcun pensiero umano. Egli ha avuto sempre davanti a Dio coscienza del peccato del mondo e ha saputo, solo lui, che realtà sia il Dio santo ed amante ${ }^{49}$.

La solitudine di Gesù, in ultima analisi, è "la solitudine di Dio nel mondo che gli

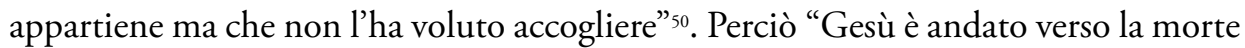
con il divino dolore, che gli uomini lo odiavano"s1. Tuttavia, pur sperimentando fino in fondo tale estrema solitudine, Gesù, mediante la sua libera-kenotica obbedienza trovava sempre nella volontà del Padre la sua vera forza e la sua vera patria. In effetti, "la volontà del Padre lo sostiene, lo ricolma, lo avvolge e spinge perennemente così che Egli, tanto solitario nel mondo, ha in essa la sua patria; così che la realizzazione d'essa è 'cibo e bevanda' per lui (Gv 4, 34)"

In definitiva, è proprio in nome della sublime solitudine del Signore che Guardini respinge una psicologia vera e propria di Gesù $\mathrm{Cristo}^{53}$ ed anche la fede di Gesù. È molto interessante, inoltre, che, colpito dall'analisi guardiniana della solitudine vissuta da Gesù, P. G. Nonis non nasconde la sua stima per Guardini e per il suo pensiero ed afferma, a ragione, al riguardo:

...pur nella sua semplicità, vien da dire ovvietà, mostra ancora una volta la straordinaria ricchezza dell'umanità guardiniana, la penetrante finezza con cui egli indaga e descrive, facendo emergere dal soggetto trattato, oltre che dall'applicazione ad esso del proprio pensiero, le note di uno dei più accattivanti e consistenti ritratti cristologici. ${ }^{54}$

\footnotetext{
${ }^{48}$ Ibid., 506.

${ }^{49}$ Ibid., 506-507.

${ }^{50}$ Idem, Il messaggio di San Giovanni: meditazioni sui testi dei discorsi dell'addio e della prima Lettera, 17.

${ }^{51}$ Ibid., 60.

52 Idem, Il Signore, 61.

${ }^{53}$ Tilliette, "La sesta beatitudine e il problema della 'coscienza di Cristo”, 30.

${ }^{54}$ Nonis, "Il Cristo di Romano Guardini”, 116.
} 


\section{La fides Christi secondo H. U. von Balthasarss}

\section{La fede biblica integrale}

Per parlare della fides Christi nella teologia contemporanea, è necessario certamente fare riferimento a diversi teologi e biblisti che si sono interessati di tale questione, sia criticandola ("contra" la fides Christi) che sostenendola ("pro" la fides Christi) 5; tuttavia, la nostra breve indagine a proposito della fede di Gesù nella teologia odierna si concentra principalmente sulla riflessione di H. U. von Balthasar ${ }^{57}$. E questo, come si è detto precedentemente, si deve alla continuità-discontinuità tra la posizione guardiniana e la posizione balthasariana; ma anche per il fatto che nella riflessione balthasariana si trovano, a nostro avviso, non soltanto una provocatoria proposta a proposito della problematica discussa, ma anche una buona sintesi biblico-storicosistematica al riguardo.

Von Balthasar, come è ben noto, ha dedicato un saggio teologico alla questione della fides Christi nella sua opera "Sponsa Verbi". La questione fondamentale di tale saggio è: Gesù stesso aveva fede? Egli credeva? Per rispondere a tale domanda, von Balthasar mette l'accento sulla "fede biblica integrale" che caratterizzava i credenti dell'antica o prima alleanza, e che è stata vissuta da Cristo in maniera archetipica. Il teologo svizzero accetta, poi, la distinzione tra il concetto di "fede" dell'Antico Testamento e quello del Nuovo Testamento, e allo stesso tempo sostiene la reale continuità tra di essi. Riferendosi a M. Buber, von Balthasar afferma che la fede veterotestamentaria sarebbe un atteggiamento integrale dell'uomo davanti a Dio, ovvero la fedeltà personale, l'abbandono assoluto, l'attesa, la fiducia... etc. (una "fede esistenziale" e un "credere in"); la fede neotestamentaria, invece, sarebbe l'accettazione di un fatto storico testimoniato dagli uomini secondo cui Gesù di Nazareth è il Cristo, il Messia, il redentore del mondo e il Figlio di Dio (una "fede dogmatica" e un "credere che"). Se la fede veterotestamentaria, però, dice von Balthasar, fosse in contrapposizione alla fede neotestamentaria, la fede del Nuovo Testamento non potrebbe essere, allora, la consumazione di quella fede dell'Antico Testamento, ma

\footnotetext{
55 A proposito del metodo teologico, delle opere e della cristologia di von Balthasar, cfr. Abdelmalak, "Haciéndose obediente hasta la muerte, y muerte de cruz", 84-98.

${ }^{56}$ Cfr., per es., Rahner, “Considerazioni dogmatiche sulla scienza e coscienza di Cristo”, 199-238; Kasper, Jesús, el Cristo, 136-137; Guillet, La fede di Gesù Cristo; Galot, "Gesù ha avuto fede?", 460-472; Sobrino, Cristología desde América Latina, 67-122; Dupuis, Introducción a la cristología, 211-213; Amato, Gesù il Signore: saggio di cristologia, 399-402; Molari, "La fede di Gesù: riflessioni sulla teologia cattolica", 53-68; Bottoni, "La fede di Gesù e la fede in Gesù nel dialogo con le altre religioni", 1-9. Per una bibliografia più ampia sulla questione, si veda Gronchi, Trattato su Gesù Cristo figlio di Dio salvatore, 1016-1017.
}

${ }^{57}$ Von Balthasar, Ensayos teológicos 2, 47-79. 
soltanto la sua sostituzione. Secondo la teologia cristiana, invece, la Nuova Alleanza è la consumazione della Antica, e il Nuovo Testamento è in continuazione con l'Antico Testamento.

In quest'ottica, la fede della nuova alleanza consuma quella dell'antica alleanza. D'altronde, per l'uomo dell'Antico Testamento, era la stessa cosa dire credere a Dio o credere alla sua parola, al suo comandamento, alla sua promessa. E per il fatto che, nel Nuovo Testamento, la Parola stessa di Dio si è fatta carne, l'accettazione da parte del credente di questa nuova ed ultima rivelazione divina non è, allora, una mutazione della stessa fede, ma è semplicemente la stessa. Si può dire, in ultima analisi, che si tratta della logica della continuità-novità, in quanto è lo stesso Dio creatore, rivelatore e salvatore, che si auto-manifesta in realtà nuova e sorprendente. Davanti a questo evento in cui il Verbo si fa carne assumendo una vera umanità, gli uomini possono credere ai annunciatori (cfr. 1Ts 2,13) della nuova alleanza (il kerygma o la fede della chiesa). Ogni uomo, se vuole far parte della nuova alleanza e del nuovo Israele, a differenza di ogni giudeo (che nasceva corporalmente come membro del popolo eletto), deve decidersi personalmente per la fides Ecclesiae e confessarla. ${ }^{58}$

\section{La fede di Gesù}

Inoltre, von Balthasar aveva cominciato il suo saggio sulla fides Christi con un "principio aprioristico" sulla fede di Gesù: se il concetto di "fede" esprime l'“atteggiamento adeguato del Popolo eletto" nei confronti di Dio, e in tale popolo di Israele, anche dell'individuo, Gesù stesso, allora, che è l'atteso di tutto l'Antico Testamento, realizza e manifesta in maniera perfetta nella sua vita tale atteggiamento richiesto da Dio Padre all'uomo. Dopo la sua riflessione sul concetto di "fede" nella Bibbia, cui si è fatto cenno sopra, il teologo svizzero ritorna ad affermare tale principio con più dettagli al riguardo.

Per lui, infatti, non è possibile che l'uomo perfetto davanti a Dio, Gesù Cristo, sia messo a confronto, con leggerezza, al compimento del vero atteggiamento dell'uomo davanti a Dio, tale come si era formato nel corso dell'Antico Testamento. Da una parte, in effetti, facendo riferimento a R. Guardini ${ }^{59}$, Egli osserva che se si parla dell'elemento specificamente neotestamentario della pistis (il "tenere come vero" il kerygma predicato), si deve dire che Cristo, che è l'oggetto essenziale di questo kerygma, non ha niente a che vedere con tale tipo di fede; e in questo senso, Cristo è al di là della fede. Dall'altra, però, per parlare della fede neotestamentaria quale consumazione interna dell'atteggiamento veterotestamentario davanti a Dio (cfr. Mt 5,17) e del modo con cui Gesù fa possibile la nostra fede, si deve parlare della fede "di" Gesù.

\footnotetext{
${ }^{58}$ Ibid., 47-54.

${ }^{59}$ Guardini, La realtà umana del Signore, 98.
} 
Questi non opera unicamente in maniera causale ciò che è stato consumato, ma lo vive lui prima, in maniera esemplare e, dopo aver ricevuto da Dio la forza redentrice, imprime in noi il suo modello vissuto ${ }^{60}$.

La tesi balthasariana, presentata sopra in forma breve, può essere riassunta, in ultima analisi, in una duplice presa di posizione di fronte alla fides Christi: se per fede si intende considerare verità tutte quelle particolari affermazioni del kerygma del Nuovo Testamento (fides quae), Gesù non aveva fede, anzi Egli è l'oggetto di tale kerygma, come diceva Guardini; ma se la parola "fede" vuole dire l'atteggiamento fondamentale del Figlio dell'uomo nei confronti di Dio, Gesù non solo aveva fede, ma anche Egli è il modello e il prototipo della fede ${ }^{61}$. In questo senso von Balthasar, a proposito la questione della fides Christi, non presenta, a nostro avviso, Gesù soltanto come la causa della nostra fede, ma anche come il suo modello. In altri termini, per lui, Gesù è la "causa-oggetto" (la fede "in" Gesù) e il "modello-prototipo" (la fede "di" Gesù) della nostra fede.

\section{L'atteggiamento centrale del Figlio dell'uomo nei confronti di Dio}

In continuazione con tutto ciò, e per chiarire bene la sua prospettiva, von Balthasar sostiene innanzitutto che il Nuovo Testamento non stabilisce nessun termine definitivo per designare l'atteggiamento centrale del Figlio dell'uomo nei confronti di $\mathrm{Dio}^{62}$. E, partendo da questo fatto, poi, cominciando dal Nuovo Testamento, passando per la patristica e la storia posteriore della teologia, e terminando con la discussione contemporanea, egli studia dettagliatamente tale difficile questione della fides Christi.

Pur avendo una perfezione suprema, umano-divina, la fede di Gesù, secondo il Nuovo Testamento, ha gli stessi elementi della fede veterotestamentaria (la fedeltà di tutto il Figlio dell'uomo al Padre e la fedeltà donata una volta per sempre e realizzata di nuovo in ogni momento del tempo). In realtà, il riferimento di tutto ciò che il Figlio è, fa e dice, al Padre e alla sua volontà è un dato chiaro nel Vangelo ${ }^{63}$. Il Nuovo Testamento contiene, poi, alcuni passaggi in cui si fa riferimento implicito alla fede

\footnotetext{
${ }^{60}$ Von Balthasar, Ensayos teológicos 2, 47,54.

${ }^{61}$ Guerriero, Hans Urs von Balthasar, 191-192.

${ }^{62}$ Von Balthasar, Ensayos teológicos 2, 55.

${ }^{63}$ In tale fedeltà di Gesù al Padre e alla sua volontà, e nella sua Kénosi, von Balthasar legge l'“ignoranza economica" del Figlio dell'uomo (Mc 13,32): "Cualquiera que sea la explicación que se dé en concreto a esta ignorancia económica del Hijo, tal ignorancia es una realidad, y ello nos basta. Esta ignorancia forma parte de su kénosis, que renuncia a muchos privilegios y posibilidades pertenecientes de jure también a la forma Dei del Hijo del Hombre" (ibid., 55). Cfr. anche Amato, Gesù il Signore, 397-399.
} 
di Gesù, che è la consumazione della fede totale-integrale dell'Antico Testamento. Nel brano di Mc 9,14-29, per es., la frase sul potere di colui che crede è, secondo von Balthasar ed altri (M. Buber), un'affermazione riferita da Gesù a se stesso. Da questo e da altri passaggi (cfr. Mt 19,17; Lc 18,19; Mt 23,23; Gv 11,41 ... etc.) si conclude che con la forza dell'abbandono di Dio in Gesù, e non solo con l'abbandono della sua soggettività da parte di Gesù al Padre, Gesù fonda la fede nei suoi discepoli, come partecipazione autentica in ciò che Gesù possiede in maniera archetipica. Solo quando si ricorda questo elemento positivo si converte la fede cristiana in fede cristiana veramente. Credere in Gesù vuole dire, allora, amare Gesù in quanto Egli è amore $\mathrm{e}$ fede in se stesso, poiché nel Verbo fatto carne si realizza la fede amante e fedele prestata a Dio e alla sua parola salvatrice dell'Antico Testamento.

La grande affermazione sulla fede di Gesù, invece, si trova nella lettera agli Ebrei dove si dice che i cristiani devono tener fisso lo sguardo eis tòn tês pisteōs archëgòn kaì teleiōtèn (Eb 12,2). Trattando il tema della fede (pisteōs), la lettera agli Ebrei parla, poi, della fede nel senso integrale dell'Antico Testamento, che si compie finalmente nel destino di Gesù: Egli è l'inizio-principio ed il perfezionatore della fede. Gesù ha combattuto previamente l'agôn della fede non soltanto in modo esemplare, bensì archetipico, rendendo possibile, dando fondamento e consumando con esso non solo la fede neotestamentaria, ma anche tutta la fede dell'Antica Alleanza ${ }^{64}$.

\section{II genitivus mysticus}

Il Nuovo Testamento, inoltre, usa una duplice formula in cui appare la parola "fede" in rapporto a Gesù: "fede di Gesù" (cfr. Gal 2,16.20; 3,22; Ef 3,12; Flp 3,9; Rm 3,22.26) e "fede in Gesù" (cfr. Gal 3,25; 5,6; Col 1,4; 2,5; Ef 1,15; 1Tm 1,14; $3,13 ; 2 \operatorname{Tm~} 1,13 ; 3,15)$. Al riguardo, la questione da risolvere è la seguente: Qual è il senso di tale duplice formula? Si tratta di un "genitivo oggettivo" o di un "genitivo soggettivo"?

Confrontandosi con tale questione, e facendo riferimento a J. Haussleitner, Gottfr. Kittel e A. Deissmann, von Balthasar afferma che il genitivo della formula "fede di Gesù" non può essere interpretato semplicemente né come un genitivus objectivus (Gesù come oggetto) né come genitivus subjectivus (Gesù come soggetto). In altri termini, non si tratta semplicemente di una fede il cui oggetto fosse Gesù, né di un atto di fede di Gesù stesso. C'è, in effetti, una terza possibilità che supera questa alternativa, ovvero il genitivus mysticus (A. Deissmann). La fede di Cristo vuol dire qui fede inquadrata nella realtà di Cristo, fede in quanto tale, partecipe della

\footnotetext{
${ }^{64}$ Von Balthasar, Ensayos teológicos 2, 55-59.
} 
pienezza di verità, amore, passione e risurrezione di Cristo, e di tutti gli altri aspetti che sono riferimento alla sua realtà. Per von Balthasar, poi, il "genitivo mistico" può essere sviluppato in due sensi: un senso, psicologico-mistico, secondo cui nella sua esperienza mistica, Paolo considera la verità della sua esistenza in Cristo e il passaggio dalla sua antica esistenza caduca in se stessa alla esistenza nuova ed autentica nella realtà di Cristo (Albert Schweitzer); ed un altro senso, oggettivo-teologico, secondo cui non si tratta soltanto della pienezza del vivere, bensì di una realtà oggettiva, a cui si orienta ed in cui si fonda l'"io" credente (Ernst Lohmeyer).

In definitiva, la "fede di Gesù" non è solo la fede che Cristo ha, neanche solo la fede che Cristo dona, ma piuttosto la fede che Egli stesso è. E tale fede è il fondamento di ogni vita di fede esistente nella storia. Tutto ciò, poi, dipende dall'unità di Cristo (l'unione ipostatica): solo per essere Dio, Cristo può convertire in principio per tutti quello che Egli stesso ha vissuto e sperimentato come morte e risurrezione; tuttavia, solo per essere uomo, poteva Cristo vivere ed esperimentare quello che è stato trasformato successivamente in principio, e si chiamerà negli altri uomini fede cristiana. Tale fede cristiana viene determinata, così, in maniera inseparabile ed egualmente chiara in un duplice atteggiamento: l'atteggiamento del Figlio nei confronti del Padre (in cui si consuma la fede di Abramo) e l'atteggiamento del Padre verso il Figlio, il quale è grazia per il mondo nella misura in cui converte l'atteggiamento del Figlio nel principio metafisico di ogni atteggiamento verso Dio. In quest'ottica, la fede paolina implica necessariamente entrambi i poli: il Gesù storico e il Cristo della fede della comunità, ovvero implica la fides Christi (la fede di Gesù e la confessione di Gesù) e la fides Ecclesiae (la fede in Gesù e la confessione del cristiano) ${ }^{65}$.

\section{Fides Dei, fides Christi e fides Ecclesiae}

In proposito della tematica in questione, ed in dialogo con il Nuovo Testamento, con la patristica e con la teologia posteriore, von Balthasar discute tanti altri aspetti importanti: la obbedienza di Gesù, la sua forma agonale o la sua lotta della fede, la relazione tra la sua fede e la sua risurrezione ${ }^{66}$, la fede e la visione (cfr. 2Cor 5,7; $\mathrm{Rm}$ 8,24; 1Cor 13,13), la spes Christi, la definizione della fede quale cognitio obscura, la

\footnotetext{
${ }^{65}$ Ibid., 59-62.

${ }^{66}$ Facendo riferimento a Ernst Fuchs, von Balthasar afferma: "Dios dio la razón, mediante la resurrección, a esta fe de Jesús, regalando con ello esta fe a la comunidad creyente como una 'fe che le favorece a esta'. Con esto quedan indicadas la cercanía y la distancia del cristianismo con respecto a Jesús: llegar a la fe significa 'situarse ante Dios juntamente con el Jesús histórico'; significa 'creer, como Jesús, que Dios escucha'. 'Pero nuestra fe se distingue de la fe de Jesús porque a nosotros se nos ha dicho, desde Pascua, en nombre de Jesús, que Dios ha escuchado”' (ibid., 64).
} 
triade inseparabile fede-carità-speranza, e la visione escatologica di Dio. La conclusione di von Balthasar, comunque, consiste nell'affermare che avere fede significa stare davanti a Dio insieme con il Gesù storico e credere come lui, anche se la fede di Gesù si distingue certamente dalla nostra, perché la nostra è in lui e grazie a lui. La fede cristiana non può intendersi separatamente dalla fede di Gesù, poiché essa è un abbandonarsi all'atteggiamento più intimo di lui. In questa prospettiva, la fede integrale vissuta da Gesù in maniera archetipica è il principio, il fondamento e la consumazione della fede degli uomini. La fides Christi si trova, così, nel crocevia vivo dell'alleanza tra Dio e il popolo eletto degli uomini. La Parola divina fatta uomo incarna in figura di uomo la fides Dei, l'alleanza fedele di Dio con l'umanità. Il Figlio di Dio umanizzato è la "fedeltà di Dio" incarnata, in cui tutte le promesse fatte da Dio trovano il loro sì (cfr. 2Cor 1,18.20).

Stando così le cose, Cristo può, come uomo perfetto, rispondere con una fede in Dio che fonda in se stessa, in maniera archetipica, tutta la fede dell'umanità; e in questo modo la fede di Gesù è l'alleanza personificata dell'umanità con Dio. Gesù Cristo è, infatti, quest'alleanza essenzialmente come "fede" nel senso integrale e come "principio metafisico" (Lohmeyer). In definitiva, il credente prende direttamente, nella fede, la fides Ecclesiae e la fides Christi, la quale è, in Cristo, ipostaticamente identica con la fides $D e i^{67}$.

\section{Obiezioni alla tesi balthasariana}

Ora, secondo J. A. Sayés, nonostante la tesi balthasariana tratta un tema che negli ultimi anni ha suscitato grande interesse nella teologia, risulta, in ultima analisi, sospetta e provoca tanti interrogativi. Partendo dalla divinità di Gesù Cristo e dalla difficoltà di conciliare tra la visione (di Dio) e la fede (in Dio), Sayes pone in discussione la tesi balthasariana ${ }^{68}$ :

Il tema della fede di Cristo è un tema di grande importanza nel quale si gioca l'identità stessa della fede cristiana: dire che "Dio mi salva perché ha convertito in principio di salvezza l'atteggiamento di fede del suo Figlio", non è lo stesso che dire "ciò che mi salva è la fede in Cristo". Questa è la specificità del cristianesimo nei confronti di tutte le altre religioni, compresa quella giudaica. Se Cristo è Dio, allora non può credere in Dio, perché questo sarebbe credere in se stesso. Se Cristo è il rivelatore del Padre in quanto il Figlio unico ed eterno, con cui condivide la stessa natura divina, allora non può credere in Dio. Non si può vedere Dio Padre nel seno della Trinità e credere in Lui. Sarebbe necessario

\footnotetext{
${ }^{67}$ Ibid., 62-79.

${ }^{68}$ Sayés, La esencia del cristianismo, 263-268.
} 
introdurre in Cristo due soggetti per fare sì che questo fosse possibile: un soggetto divino e un soggetto umano. ${ }^{69}$

A giudizio di Sayés, poi, ci sono tre fondamentali obiezioni contro la tesi balthasariana: il fondamento biblico, i testi che parlano della fede di Gesù e la relazione tra l'ontologia di Gesù e la sua psicologia. Come prima obiezione, si deve riconoscere che nella Scrittura, eccetto il testo di (Eb 12,2), non solo non esiste un testo che dica che Gesù crede, anzi in essa Cristo esige per se stesso la fede che esige per il Padre. Il testo di (Eb 12,2), poi, deve essere inteso non nel senso di una fede soggettiva di Cristo, ma come iniziatore e consumatore della nostra fede grazie alla sua incarnazione e risurrezione. A proposito della seconda obiezione, inoltre, i testi che parlano della fede di Gesù non indicano Cristo come soggetto, bensì come termine della nostra fede in Cristo (in dativo). In altre parole, la fede di Gesù non è un atto di Cristo, ma un atto di colui che crede in Cristo, poiché il soggetto di tali testi non è Gesù, ma l'uomo che crede in Lui. Con l'ultima obiezione, la relazione tra l'ontologia di Gesù e la sua psicologia, si vuole dire che l'unica prospettiva valida è quella che presenta il Verbo come soggetto della visione del Padre (nella natura divina) e questo stesso Verbo come testimone in parole umane, testimone nella sua umanità. Mentre l'umanità di Cristo è una umanità in kénosi, si potrebbe accettare che Cristo, come uomo, confidava nel Padre e si abbandonava a lui. Ma questo non significa che Egli aveva fede nel Padre ${ }^{70}$.

\section{Una breve conclusione}

In realtà, la questione della fides Christi (cfr. Eb 12,2) e dell'ubbidienza di Cristo (cfr. Eb 5,7-10) pare una questione teologica ancora aperta. A nostro avviso, però, sembra più giusto e più biblico parlare principalmente, come fa giustamente Guardini, di un'ubbidienza di Gesù piuttosto che di una fede di Gesù. Nella sua condizione di Figlio di Dio pienamente umanizzato, cioè, Gesù obbediva fiduciosamente e si affidava obbedientemente a suo Padre. In quest'ottica, nelle seguenti parole della Pontificia Commissione Biblica si trova una buona sintesi che riflette l'interpretazione guardiniana a proposito dell'obbedienza filiale-kenotica di Gesù.

Il segreto ultimo -o piuttosto il mistero- di Gesù consiste essenzialmente nel suo rapporto con Dio. Nella sua preghiera Egli lo chiama "Abba": parola che in aramaico significa "Padre" con una sfumatura di familiarità (cfr. Mc 11,36 ecc.). Nella medesima frase Egli si attribuisce il nome di "Figlio" in cui afferma che solo il Padre conosce il giorno del Giudizio, escludendo gli Angeli e il

\footnotetext{
${ }^{69}$ Ibid., 268. La traduzione è nostra.

${ }^{70}$ Ibid., 269-271.
} 
Figlio stesso (Mc 13,32) [...]. Questo è il segreto intimo in cui trovano la loro origine tutti i comportamenti di Gesù, oppure, per esprimersi in altri termini, la sua autentica filialità (o condizione filiale). Egli ha coscienza di ciò fin dalla fanciullezza (Lc 49) e lo manifesta con la sua perfetta obbedienza alla volontà del Padre (Mc 14,36 e par.). Questa condizione di Figlio non gli impedisce di essere un uomo in modo perfetto, che "cresceva in sapienza, età e grazia davanti a Dio e agli uomini" (Lc 2,52). Così egli cresce acquisendo una coscienza sempre più precisa della missione affidata a Lui dal Padre, dall'infanzia fino alla morte in croce. ${ }^{71}$

È possibile sostenere, dunque, che, per il fatto che non abbiamo tanti testi chiari in riferimento alla questione della fede di Gesù (Gesù come "soggetto" della fede), sarebbe più adeguato, come affermano diversi teologi odierni ${ }^{72}$, parlare dell'obbedienza di Gesù, appunto a causa della chiarezza dei testi della Scrittura al riguardo, e non della fede di Gesù ${ }^{73}$.

Né nei vangeli -afferma A. Amato-, né nel kerygma primitivo ci sono testimonianze che fondano l'esistenza della fede da parte di Gesù Cristo. Gesù ama, vede, conosce, prega, invoca il Padre, gli obbedisce. Egli, però, non "crede" nel Padre, appunto perché è in intima e costante unione di visione con lui. Egli pretende dagli altri la fede nel Padre e in se stesso (cfe. Gv 14,1). ${ }^{74}$

L'obbedienza filiale-kenotica di Gesù è, infatti, una obbedienza fiduciosa nei confronti del suo Padre. Tuttavia, per affermare la vera umanità di Gesù e la sua autentica esperienza religiosa personale, come vero uomo la cui disposizione fondamentale davanti al suo Padre amoroso è quella della fiducia e dell'abbandono, ci si deve, a nostro avviso, interrogare seriamente, come pensa giustamente von Balthasar, sul suo atteggiamento integrale nei confronti del suo Padre. E ciò per le ragioni seguenti:

1. Parlare dell'obbedienza di Gesù implica necessariamente un certo tipo di fede (l'obbedienza di fede), perché non c'è e non può esserci una vera-libera obbedienza senza fede (fides qua).

2. La fides Christi, nel senso di fides qua (credere in), e non nel senso di fides quae (credere che), esprime la fedeltà di Dio (fides Dei) e fonda la fede della Chiesa (fides Ecclesiae).

\footnotetext{
${ }^{71}$ Pontificia Commissione Biblica, Bibbia e cristologia, 99.101.

72 Amato, Gesù il Signore, 399-402; Gronchi, Trattato su Gesù Cristo figlio di Dio salvatore, 1038-1040; ibid., 1039: "Da parte nostra, riteniamo che sia più conveniente parlare di 'fiduciosa obbedienza' di Gesù -tema ben più attestato dal punto di vista neotestamentario-, per esprimere adeguatamente la crescita e maturazione nella sua condizione filiale (cfr. Eb 5,8), in prospettiva della assunzione dell' humanum". ${ }^{73}$ Tale argomento era anche quello di San Tommaso. Al riguardo, cfr. Santo Tomás de Aquino, Suma de teología, Tomo V, p. III, q. 7, a. 3; Von Balthasar, Ensayos teológicos 2, 70.
}

${ }^{74}$ Amato, Gesù il Signore, 339. 
3. La fede di Gesù è certamente uno dei più importanti elementi del vero ed autentico umanizzarsi del Figlio di Dio davanti a Dio, il suo Padre, e davanti agli uomini, i suoi fratelli.

4. Gesù non è soltanto la "causa" della fede (causa efficiente), ma è anche il suo esempio e il suo "modello archetipico" (causa esemplare).

In questo senso la fede che ci salva e giustifica, infatti, non è semplicemente la nostra fede in Gesù Cristo, ma è essenzialmente la fede di colui è l'iniziatore, l'autore,

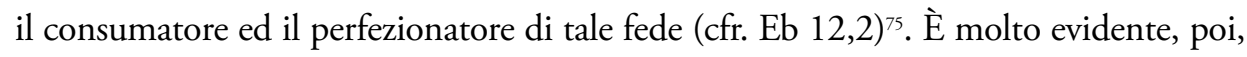
che si deve certamente distinguere tra la nostra fede in Gesù e la fede di Gesù, perché la nostra fede è causata dalla fede di Gesù Cristo, dalla fede del Figlio di Dio pienamente umanizzato. Perciò, come dice G. Bottoni,

La fede in Gesù (e pertanto nella sua divinità) da parte dei cristiani, se non è supportata da una loro lucida e corretta consapevolezza della sua fede (fede di Gesù), è esposta al noto e più volte denunciato rischio di una cristologia tendenzialmente monofisita. Tale tendenza, anche se in modo non voluto e del tutto inconscio, è assai diffusa nella pietà cristiana, che spesso, in alternativa al mancato ruolo salvifico dell'umanità di Gesù, si è dovuta cercare le necessarie mediazioni umane in varie forme di devozione e devozionismi. ${ }^{76}$

In definitiva, la teologia sistematica ha la possibilità ed il compito di riscoprire la vera ed autentica umanità di Gesù di Nazareth e la sua autentica esperienza spiritualereligiosa personale anche nel campo della fede; poiché la fede "di" Gesù di Nazareth, il Figlio di Dio ed il Figlio dell'uomo, è il "fondamento fondante" della fede ecclesiale e personale "in" Gesù il Cristo. Nelle nostre future riflessioni, ci accingeremo a sviluppare questa proposta teologico-sistematica.

\section{Bibliografia}

Abdelmalak, Ashraf N. I. "Haciéndose obediente hasta la muerte, y muerte de cruz": R. Guardini y H. U. von Balthasar ante el sentido teológico de la muerte en cruz de Jesucristo. Bogotá: Editorial Bonaventuriana, 2017.

Alszeghy, Zoltan; Maurizio Flick. Come si fa teologia: introduzione allo studio della teologia dogmatica. Alba: Edizioni Paoline, 1974.

Amato, Angelo. Gesù il Signore: saggio di cristologia. Bologna: Edizioni Dehoniane, 1988.

\footnotetext{
${ }^{75}$ Guillet, La fede di Gesù Cristo, 179.

${ }^{76}$ Bottoni, "La fede di Gesù e la fede in Gesù nel dialogo con le altre religioni”, 1.
} 


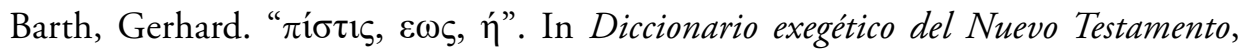
G. Schneider; H. Balz (edd.), Vol. II, 942-961. Salamanca: Sígueme, 2012.

Bottoni, Gianfranco. "La fede di Gesù e la fede in Gesù nel dialogo con le altre religioni (2008)", 1-9. Decanato de Monza, http://www.decanatomonza.it/files/ scuola\%20teologia/XVII/Bottoni.pdf (consultado el 15 de diciembre de 2016).

Bueno De la Fuente, Eloy. "Guardini, Romano". In Diccionario de teólogos/as contemporáneos, Juan Bosch Navarro (ed.), 460-471. Burgos: Monte Carmelo, 2004.

Dheilly, Joseph. "Fe". In Diccionario biblico, J. Dheilly (ed.), 445-453. Barcelona: Herder, 1970.

Duplacy, Jean. "Fe". In Vocabulario de teología bíblica, X. Léon-Dufour et allii (edd.), 327-335. Barcelona: Herder, 1980.

Dupuis, Jacques. Introducción a la cristología. Estella (Navarra): Editorial Verbo Divino, 2000.

Engelmann, Henri; Francis Ferrier. "Introduzione a Romano Guardini". Giornale di Teologia 22 (1968).

Francesco I. "Lettera enciclica Lumen fidei (29 giugno 2013)". Vatican, http://w2.vatican.

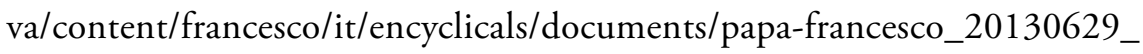
enciclica-lumen-fidei.html (consultato il 10 di marzo 2014).

Galot, Jean. “Gesù ha avuto fede?”. La civiltà cattolica 103 (1982): 460-472.

Gerl, Hanna-Barbara. Romano Guardini: La vita e l'opera. Brescia: Morcelliana, 1988.

Gerstenberger, Erhard. "Confiar". In Diccionario teológico manual del Antiguo Testamento, E. Jenni; C. Westermann (edd.), Tomo I, 439-446. Madrid: Cristiandad, 1978.

Gibellini, Rosino. La teología del siglo XX. Santander: Sal Terrae, 1998.

González De Cardedal, Olegario. "La obra teológica de Hans Urs von Balthasar". Communio, ed. española, N. ${ }^{\circ}$ IV (1988): 365-396.

Gronchi, Maurizio. Trattato su Gesù Cristo figlio di Dio salvatore. Brescia: Queriniana, 2012.

Guardini, Romano. Apuntes para una autobiografía. Madrid: Encuentro, 1992. . Fede-religione-esperienza: saggi teologici. Brescia: Morcelliana, 1984. . Il messaggio di San Giovanni: meditazioni sui testi dei discorsi dell'addio e della prima lettera. Brescia: Morcelliana, 1982. 
. Il Signore: Riflessioni sulla persona e sulla vita di Gesù Cristo. Brescia: Vita e Pensiero-Morcelliana, 2005.

. Jesucristo: palabras espirituales. Madrid: Cristiandad, 1965.

. L'esistenza e la fede. Brescia: Morcelliana, 1960.

. La fe en nuestro tiempo. Madrid: Cristiandad, 1965.

. La figura di Gesù Cristo nel Nuovo Testamento. Brescia: Morcelliana, 2000.

. La realtà umana del Signore: saggio sulla psicologia di Gesù. Brescia: Morcelliana, 1970.

. La vita della fede. Brescia: Morcelliana, 2008.

. Libertad, gracia y destino. San Sebastian: Dinor, 1954.

Guerriero, Elio. Hans Urs von Balthasar. Madrid: San Pablo, 2008.

Guillet, Jacques. La fede di Gesù Cristo. Milano: Jaca Book, 1982.

Hadjadj, Fabrice. La fede dei demoni: Ovvero il superamento dell'ateismo. GenovaMilano: Casa Editrice Marietti, 2010.

Henrici, Peter. "Semblanza de Hans Urs von Balthasar". Communio, ed. española, N. ${ }^{\circ} \operatorname{IV}-V(1989): 356-391$.

Kasper, Walter. Jesús, el Cristo. Salamanca: Sígueme, 1978.

Lehmann, Karl; Walter Kasper (edd.). Hans Urs von Balthasar: figura e opera. Casale Monferrato: Piemme, 1991.

López Quintás, Alfonso. "Estudio introductorio”. In Ética: lecciones en la Universidad de Múnich, R. Guardini, xvii-xxxiii. Madrid: Biblioteca de Autores Cristianos, 2000.

. La verdadera imagen de Romano Guardini. Pamplona: Eunsa, 2001.

. Romano Guardini: maestro de vida. Madrid: Palabra, 1998.

Marconcini, Benito. "Fe". In Nuevo diccionario de teología bíblica, P. Rossano; G. Ravasi; A. Girlanda (edd.), 653-671. Madrid: Paulinas, 1990.

Molari, Carlo. "La fede di Gesù: riflessioni sulla teologia cattolica". In AA. VV., "Se aveste fede quanto un granello di senape... ": Atti della XLII Sessione di Formazione Ecumenica, 53-68. Milano: Ancora, 2006.

Mondin, Battista. I grandi teologi del secolo ventesimo. Vol. 1. I teologi cattolici. Torino: Borla Editore, 1972. 
. La nuova teologia cattolica: da Karl Rahner a Urs von Balthasar. Roma: Edizioni Logos, 1978.

Nieto, Evaristo Martín. "Fe". In Diccionario de Jesús de Nazaret, F. F. Ramos (ed.), 436-442. Burgos: Monte Carmelo, 2007.

Nonis, Pietro Giacomo. "Il Cristo di Romano Guardini". In La Weltanschauung cristiana di Romano Guardini, a cura di S. Zucal, 107-124. Bologna: Centro Editoriale Dehoniano, 1988.

Pontificia Commissione Biblica. Bibbia e cristologia. Cinisello Balsamo (MI): Edizioni Paoline, 1987.

Rahner, Karl. "Considerazioni dogmatiche sulla scienza e coscienza di Cristo". In idem, Saggi di cristologia e di mariologia, 199-238. Roma: Edizioni Paoline, 1965.

Santo Tomás de Aquino. Suma de teología. Tomo III e Tomo V. Madrid: Biblioteca de Autores Cristianos, 2010.

Sayés, José Antonio. La esencia del cristianismo: diálogo con K. Rahner y H. U. von Balthasar. Madrid: Cristiandad, 2005.

Scola, Angelo. Hans Urs von Balthasar: un estilo teológico. Madrid: Encuentro, 1997.

Sobrino, Jon. Cristología desde América Latina. México: CRT, 1976.

Sommavilla, Guido. "Introduzione a R. Guardini". In Scritti filosofici. A cura di G. Sommavilla, Vol. 1, 123-132. Milano: Fratelli Fabbri Editori, 1964.

Tilliette, Xavier. "La sesta beatitudine e il problema della 'coscienza di Cristo"'. Communio 102 (1988): 27-36.

Von Balthasar, Hans Urs. Ensayos teológicos. Vol. 2: Sponsa Verbi. Madrid: CristiandadEncuentro, 2001. . Romano Guardini: riforma dalle origini. Milano: Jaca Book, 2000.

Wildberger, Hans. "Firme, seguro". In Diccionario teológico manual del Antiguo Testamento, E. Jenni; C. Westermann (edd.), Tomo I, 276-319. Madrid: Cristiandad, 1978. 